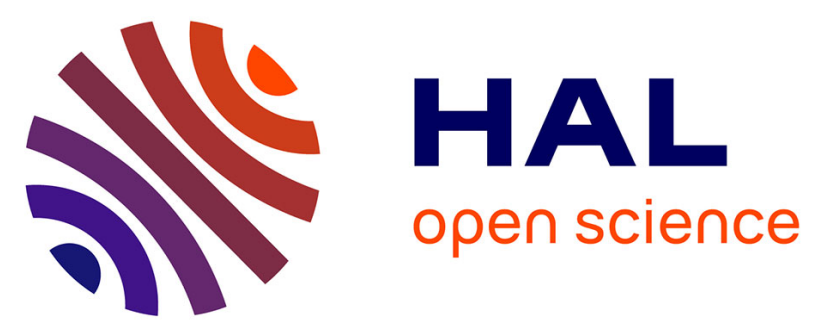

\title{
Influence of hole depletion and depolarizing field on the BaTiO3/La0.6Sr0.4MnO3 interface electronic structure revealed by photoelectron spectroscopy and first-principles calculations
}

D.G. Popescu, Nicholas Barrett, Christina Chirila, Iuliana Pasuk, M. A.

Husanu

\section{To cite this version:}

D.G. Popescu, Nicholas Barrett, Christina Chirila, Iuliana Pasuk, M. A. Husanu. Influence of hole depletion and depolarizing field on the BaTiO3/La0.6Sr0.4MnO3 interface electronic structure revealed by photoelectron spectroscopy and first-principles calculations. Physical Review B: Condensed Matter and Materials Physics (1998-2015), 2015, 92, pp.235442. 10.1103/PhysRevB.92.235442 . cea01347245

\author{
HAL Id: cea-01347245 \\ https://hal-cea.archives-ouvertes.fr/cea-01347245
}

Submitted on 20 Jul 2016

HAL is a multi-disciplinary open access archive for the deposit and dissemination of scientific research documents, whether they are published or not. The documents may come from teaching and research institutions in France or abroad, or from public or private research centers.
L'archive ouverte pluridisciplinaire HAL, est destinée au dépôt et à la diffusion de documents scientifiques de niveau recherche, publiés ou non, émanant des établissements d'enseignement et de recherche français ou étrangers, des laboratoires publics ou privés. 


\title{
Influence of hole depletion and depolarizing field on the $\mathrm{BaTiO}_{3} / \mathrm{La}_{0.6} \mathrm{Sr}_{0.4} \mathrm{MnO}_{3}$ interface electronic structure revealed by photoelectron spectroscopy and first-principles calculations
}

\author{
Dana G. Popescu, ${ }^{1}$ Nicholas Barrett, ${ }^{2}$ Cristina Chirila, ${ }^{1}$ Iuliana Pasuk,,${ }^{1}$ and Marius A. Husanu ${ }^{1, *}$ \\ ${ }^{1}$ National Institute of Materials Physics, Atomistilor 105bis, 077125 Magurele-Ilfov, Romania \\ ${ }^{2}$ Service de Physique de l'Etat Condens, DSM/IRAMIS/SPEC, CNRS UMR 3680, CEA Saclay, 91191 Gif sur Yvette cedex, France
}

(Received 13 August 2015; published 28 December 2015)

\begin{abstract}
The effects of the bonding mechanism and band alignment in a ferroelectric (FE) $\mathrm{BaTiO}_{3} /$ ferromagnetic $\mathrm{La}_{0.6} \mathrm{Sr}_{0.4} \mathrm{MnO}_{3}$ heterostructure are studied using $\mathrm{x}$-ray photoelectron spectroscopy and first-principles calculations. The band lineup at the interface is determined by a combination of band bending and polarization-induced modification of core-hole screening. A Schottky barrier height for electrons of $1.22 \pm 0.17 \mathrm{eV}$ is obtained in the case of downwards FE polarization of the top layer. The symmetry of the bonding states is emphasized by integrating the local density of states $\pm 0.2 \mathrm{eV}$ around the Fermi level, and strong dependence on the FE polarization is found: upwards, polarization stabilizes Ti $t_{2 g}(x y)$ orbitals, while downwards, polarization favors Ti $t_{2 g}(y z)$ symmetry. It is predicted that the abrupt $(\mathrm{La}, \mathrm{Sr}) \mid \mathrm{TiO}_{2}$ interface is magnetoelectrically active, leading to a A-type antiferromagnetic coupling of the first $\mathrm{TiO}_{2}$ interface layer with the underlying manganite layer through a superexchange mechanism.
\end{abstract}

DOI: 10.1103/PhysRevB.92.235442

PACS number(s): 73.20.-r, 72.25.-b, 77.55.fe, 77.80.bn

\section{INTRODUCTION}

The electronic origin of the magnetoelectric coupling at a ferroelectric/ferromagnetic (FE/FM) interface where the bottom FM electrode is a hole-doped manganite $(\mathrm{Ca}, \mathrm{Sr})$, $\mathrm{LaMnO}_{3}$, has been recently addressed in a number of papers [1-9]. It is relatively well understood that screening charges are pulled towards the interface from the FM layer, leading to hole depletion when the FE polarization points towards the interface and hole accumulation when FE polarization points away from the interface. This, in turn, can be reflected in the modulation of the electroresistance depending on the FE polarization direction [1], opening the way, for example, for resistive readout in random access memories based on FE/FM tunnel junctions.

If one takes into account the electron spin, functionality can be extended to four-state memories and to controlled, spin-dependent current injection into an insulating layer sandwiched between the ferromagnet electrodes. Therefore, combining ferroelectricity with additional degrees of freedom offers opportunities for developing interesting applications in, for example, spin-dependent transport [9-12] or devices where magnetocrystalline anisotropy is controlled by electric fields [13].

A prerequisite for the efficient functioning of such devices is detailed knowledge of the mechanisms that determine the electronic behavior of the electrode/FE interface and the nature of the coupling of the spin and ferroelectricity.

Hole-doped FM $\mathrm{La}_{1-x} \mathrm{Sr}_{x} \mathrm{MnO}_{3}$ (LSMO) exhibits a very rich phase diagram depending on the hole doping level $x$, including metal-insulator transition and the colossal magnetoresistance $(\mathrm{CMR})$ effect accompanied by transition from the A-type antiferromagnetic $(x \sim 0.5)$ [14] to FM states $[15,16]$. For the case of a FM buried under a FE, when the FE polarization of the top layer is out of plane, it was also shown that depletion or accumulation of holes which is induced at the FE/FM interface leads to changes in the orbital order [17],

*ahusanu@infim.ro suggesting strong interplay between the spin, charge, lattice, and orbital degrees of freedom in FE/FM heterostructures.

When dealing with very thin interfaces of only a few lattice constants, transport measurements become difficult, if not impossible, because of high leakage currents. In photoemission experiments this shortcoming is not encountered, allowing for direct inspection of the interface electronic structure. However, when dealing with FE surfaces or interfaces, subtle binding energy shifts may arise from the screening of the polarization charges by the photogenerated carriers.

For example, in a recent study performed with synchrotron soft $x$ rays aiming to reveal interface effects in a metal/FE heterojunction [LSMO/PbZr $\left.{ }_{x} \mathrm{Ti}_{1-x} \mathrm{O}_{3}(\mathrm{PZT})\right]$, Wu et al. [18] reported a rapid variation in time of the binding energy at the bottom interface, obeying a typical rate equation for diffusion. Standard laboratory $\mathrm{x}$-ray sources, on the other hand, deliver much lower flux than synchrotron beam lines; thus, direct access at the interface states is possible.

The depolarization field in the FE, which tends to restore the local neutrality of the system, may be fully or partially screened by the metallic electrodes. This leads in turn to subtle variations of the internal field which influence the position of the x-ray photoelectron spectroscopy (XPS) peaks of the $\mathrm{BaTiO}_{3}$ (BTO) and induce peculiar variations of photoemission features [19-24].

In this context, our paper presents experimental results on interface doping, Schottky barrier height, and carrier accumulation/depletion obtained by soft $\mathrm{x}$-ray photoemission and compares them with first-principles calculations for the buried interface of LSMO $(x=0.4)$ under a thin ferroelectric top layer - BTO.

The structure of this paper is as follows: Sec. II presents the sample preparation and experimental details; Sec. III deals with the details of the $a b$ initio calculations. The experimental results will be presented in Sec. IV A for structure and morphology, in Sec. IV B for XPS data, and in Secs. IV C and IV D for Schottky barrier height and interface charge anisotropy, followed by the computational data obtained with first-principles calculations in Sec. V. 


\section{EXPERIMENT}

Two samples were prepared: a single-crystal 30-nm-thick $\mathrm{La}_{1-x} \mathrm{Sr}_{x} \mathrm{MnO}_{3}$ film, $x=0.4$, grown by pulsed laser deposition (PLD) on $\mathrm{SrTiO}_{3}$ (100) (STO) and $\sim 5$-nm-thick $\mathrm{BaTiO}_{3}$ on top of the same LSMO/STO structure. The PLD setup (Surface $\mathrm{GmbH}$ ) uses $\mathrm{KrF}$ radiation (248-nm wavelength). Samples were prepared by using pulses of 0.7-J energy with a duration of $20 \mathrm{~ns}$. The repetition rate was $5 \mathrm{~Hz}$, and the laser fluence was $2-3 \mathrm{~J} / \mathrm{cm}^{2}$. During the LSMO growth, the substrate was heated to $800^{\circ} \mathrm{C}$, and the partial $\mathrm{O}_{2}$ pressure was $0.2 \mathrm{mbar}$. The samples were introduced afterwards in the XPS chamber. Using the same conditions, a 5-nm-thick BTO film was deposited on the LSMO films at $700{ }^{\circ} \mathrm{C}$ in flowing $\mathrm{O}_{2}$, and the growth was monitored by reflection high-energy electron diffraction (RHEED), which confirmed the layer-by-layer growth. In this manner single-crystal thin-film heterostructures with abrupt interfaces are achieved [24].

Photoelectron spectroscopy measurements were conducted in a dedicated chamber equipped with a monochromatized x-ray source XM $1000 \mathrm{MkII}\left(\mathrm{Al} \mathrm{K} \mathrm{K}_{\alpha}: h v=1486.7 \mathrm{eV}\right.$ ) and a SPHERA II analyzer, both from Omicron Nanotechnology $\mathrm{GmbH}$. A pass energy of $40 \mathrm{eV}$ was used, along with a step of $0.1 \mathrm{eV}$. The pressure was kept below $7.2 \times 10^{-10} \mathrm{mbar}$, and the energies were calibrated to the $\mathrm{C} 1 \mathrm{~s}$ spectrum. The core-level spectra were fitted with Voigt lines, associated integrals of the Voigt profile [25], and different background lines associated with the inelastic scattering of photoelectrons. This permits simulating a multicomponent spectrum using different background terms for each component in order, for example, to assess the location of the emitter with respect to the surface, instead of employing a single Shirley background for the entire spectrum [26].

The relative position of the light source and the analyzer are fixed; the emission angle is controlled by rotating the samples. XPS spectra were recorded for two orientations of the electron detector with respect to the sample in order to vary the depth sensitivity: normal to the surface and at $60^{\circ}$. The crystallinity was investigated by $\mathrm{x}$-ray diffraction (XRD) and low-energy electron diffraction (LEED).

Before XPS analysis, both samples were annealed at $250-300{ }^{\circ} \mathrm{C}$ in UHV for $2 \mathrm{~h}$ in order to remove as much of the residual contamination of the surface as possible.

\section{COMPUTATIONAL DETAILS}

Calculations have been performed within the density functional theory (DFT) framework as implemented in the QUANTUM ESPRESSO code [27]. The code treats the atoms with ultrasoft pseudopotentials [28] and considers explicitly valence and semicore electrons, using plane waves to expand the multielectronic wave function. The exchange and correlation contributions to the total Hamiltonian are treated within the generalized gradient approximation in the formulation of Perdew and Wang plus on-site Coulomb interaction $(\mathrm{GGA}+U, \mathrm{PW} 91)$ [29]. The atomic valence configuration, including semicore states used in the calculations, is Ti, $3 s^{2} 3 p^{6} 3 d^{1} 4 s^{2} 4 p^{0}$; $\mathrm{Mn}, 3 s^{2} 3 p^{6} 3 d^{5} 4 s^{2} 4 p^{0}$; $\mathrm{Ba}$, $5 s^{2} 5 p^{6} 5 d^{0} 6 s^{2} 6 p^{0} ; \mathrm{O}, 2 s^{2} 2 p^{4}$. For Mn atoms a $U_{\mathrm{Mn}}=2 \mathrm{eV}$ Hubbard parameter was employed, which reproduces well the

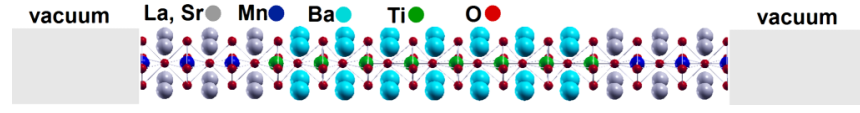

FIG. 1. (Color online) Model of a symmetric supercell as used in calculations, with LSMO contacts at both ends, separated by $20 \AA$ of vacuum

half-metallic character of the LSMO [24,30], while in the case of Ti atoms, $U_{\mathrm{Ti}}=8 \mathrm{eV}$ was used, which, on the one hand, reduces the overlap of the $3 d$ orbitals and, on the other, solves the problem associated with the underestimation of the BTO band gap. The band gap of bulk BTO for $U_{\mathrm{Ti}}=0 \mathrm{eV}$ was $0.84 \mathrm{eV}$, whereas using $U_{\mathrm{Ti}}=8 \mathrm{eV}$, it became $2.14 \mathrm{eV}$, closer to the experimental value of $3.2 \mathrm{eV}$ [31]. This is important for the valence-band alignment and in calculating the Schottky barrier height; otherwise, results could be misinterpreted due to unrealistic charge-transfer effects between the metallic contact and the insulator [32].

The BTO bulk polarization, $P=26 \mu \mathrm{C} / \mathrm{cm}^{2}$, was calculated using Berry's phase method [33] for the two configurations minimizing the total energy, denoted $P^{+}$and $P^{-}$. The former case implies a positive relative displacement of the central $\mathrm{Ti}$ atom with respect to the adjacent oxygen atoms along the [001] direction and negative displacement in the latter case. Assuming epitaxial growth, confirmed by $\mathrm{XRD}$, the heterostructure was modeled in $1 \times 1$ slab geometry as periodically repeated supercells starting from the relaxed structure of the LSMO unit cell but imposing the calculated in-plane lattice constant $a=3.903 \AA$ of $\mathrm{SrTiO}_{3}$. It contains a central 8-unit-cell (u.c.) BTO slab in contact with a 5-u.c. LSMO electrode at either end. In this manner, an electron reservoir is ensured at both ends of the ferroelectric in order to correctly compensate the polarization charges from the FE slab. Then, the coordinates were allowed to relax along the $z$ axis only until the Hellmann-Feynman forces on each atom were less than $30 \mathrm{meV} / \AA$. La-Sr substitutional doping was treated by means of a virtual crystal approximation (VCA) replacing each A site of the perovskite with a fictitious atom with fractional valence, instead of explicit doping, which would require a significant computational burden due to larger supercells. Calculations were performed using a $30 \mathrm{Ry}$ cutoff for the plane-wave expansion and $400 \mathrm{Ry}$ for the chargedensity integration. A $5 \times 5 \times 1 k$-point mesh was used for relaxation of coordinates in the supercell in the $z$ direction, and a denser $10 \times 10 \times 1$ grid was used for calculating the density of states and charge distribution in the interface regions.

Supercells were separated by $20 \AA$ of vacuum in order to prevent the interaction between periodically repeated slabs (Fig. 1). This geometry allows us to obtain in a single calculation the relevant electronic quantities of both electrode $/ P^{+}$ and electrode $/ P^{-}$interfaces for the out-of-plane direction of the ferroelectric polarization. It also avoids the well-known issue of how to realistically screen free ferroelectric surfaces in finite supercells [34]. Of course, this approach does not offer information on the relaxation mechanisms, rumpling, or other surface effects, but within this paper we are mostly concerned with the interface properties, and our treatment gives insight into the LSMO/BTO interface region $[6,7,30]$. 


\section{EXPERIMENTAL RESULTS}

\section{A. Structure and morphology}

Figure 2(a) presents the x-ray diffraction pattern of the BTO/LSMO/STO heterostructure. The LSMO layers present a rhombohedral structure with in-plane parameter $a=5.5212 \AA$ and out-of-plane parameter $c=13.3797 \AA$ and hence a $c / a=$ 2.4233 ratio. The LSMO film thickness determined by $\mathrm{x}$-ray reflectivity is $30 \mathrm{~nm}$. Atomic force microscopy (AFM) on the LSMO bottom layer confirms the presence of smooth terraces, which implies a chemically homogeneous interface between BTO layers and the substrate (LSMO) over the whole sample.

The LSMO peaks were indexed according to the rhombohedral LSMO structure (space group $R-3 c$ ); the data indicate a (012) texture for LSMO and a (001) orientation for BTO. The apparition of layer fringes (Fs) around the (012) LSMO peak indicates a sharp BTO/LSMO interface. If the ratio $c / a$ of a rhombohedral lattice in hexagonal notation is 2.45 , then the angle of the primitive rhombohedral cell is $60^{\circ}$, and the lattice can be described as fcc. For our LSMO the ratio is 2.42, so the lattice symmetry is close to cubic [35]. The relationship between the hexagonal lattice plane indices and the cubic ones is such that $(012)_{\text {hex }}$ corresponds to (002) cubic $_{\text {. Thus, LSMO-r }}$ with (012) texture can be described as pseudocubic LSMO with (001) texture with a lattice constant $a=3.89 \AA$, subjected to a tensile in-plain strain, which leads to a compressive strain normal to the surface. Figure 2(b) shows an AFM image recorded on a bare LSMO surface, revealing smooth

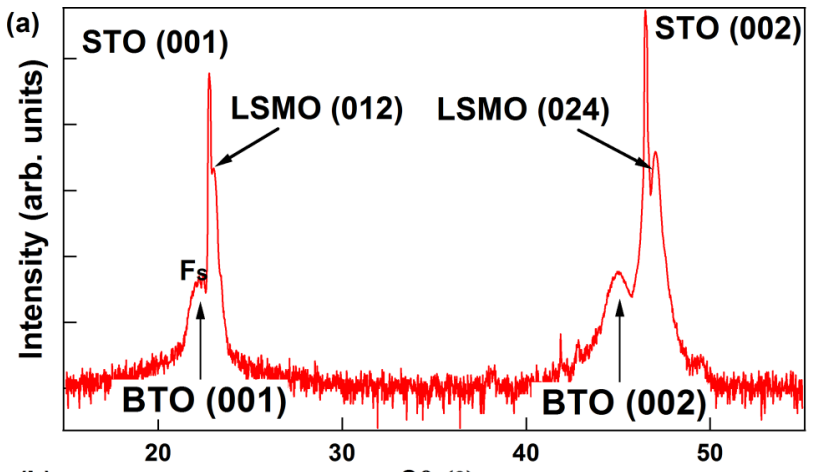

(b) $2 \theta\left({ }^{\circ}\right)$

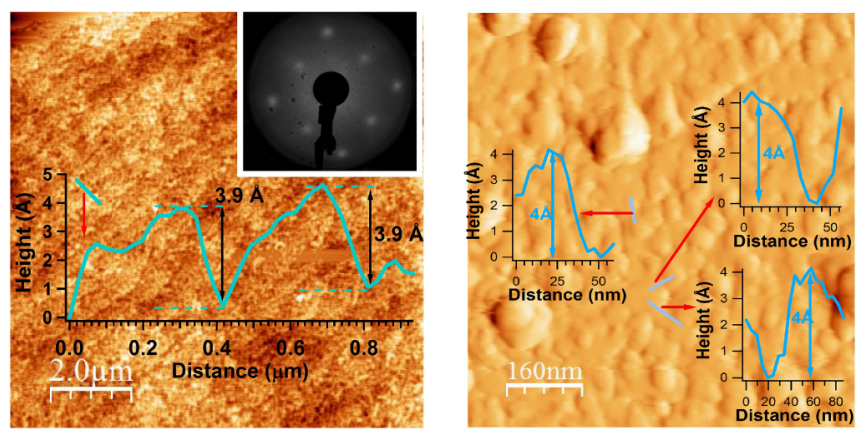

FIG. 2. (Color online) (a) XRD diffraction profile on a BTOcovered LSMO layer. (b) An AFM image of the bare LSMO sample showing the large-area terrace with single-unit stepping height and $(1 \times 1)$ LEED pattern. (c) An AFM image recorded on a BTO-covered LSMO sample showing the uniform character of the resulting surface and the single unit-cell steps between most of the surface features. terraces, and the inset shows the $(1 \times 1)$ LEED pattern of the LSMO surface. The AFM recorded on the BTO-covered LSMO sample [Fig. 2(c)] confirms the large-area smoothness of the resulting surface. The topography of the BTO surface [Fig. 2(c)] reveals continuous coverage of the LSMO substrate and a step height of $\sim 4 \AA$ between different protrusions along the lines marked in blue, suggesting a single surface termination of the BTO layer [36].

\section{B. X-ray photoelectron spectroscopy}

As already stated, dealing with such a thin ferroelectric in transport measurements is cumbersome due to leakage currents at the interface, and extracting the relevant parameters of the heterostructure, such as conductivities or Schottky barrier heights (SBHs), is intricate work. The direct way of addressing the SBH in photoelectron spectroscopy, on the other hand, is based on inferring the band alignment at the interface from the position of the core-level peaks with respect the valence-band maximum (VBM) in the insulator and the Fermi level in the metal.

For the case of an interface where a ferroelectric is involved, the SBH contains the effects of both band bending and depolarizing field, on the one hand, and, on the other hand, the intrinsic electronic contribution of the lineup at the interface. Thus, the height of the Schottky barrier, accounting for both the effects of depolarizing field and band bending [21,37,38], can be written as the sum

$$
\Phi_{p}=\Delta E_{v}+\Delta V(z)
$$

where $\Delta V(z)$ stands for ferroelectric-induced effects and $\Delta E_{v}$, the valence-band offset term, which has exclusively electronic origin, can be expressed as follows $[18,39,40]$ :

$$
\begin{aligned}
\Delta E_{v}= & \left(E_{\mathrm{Ti} 2 \mathrm{p}}-E_{\mathrm{Sr} 3 \mathrm{~d}}\right)_{\mathrm{LSMO} / \mathrm{BTO}}-\left(E_{\mathrm{Sr} 3 \mathrm{~d}}-E_{\mathrm{F}}\right)_{\mathrm{LSMO}} \\
& \times\left(E_{\mathrm{Ti} 2 \mathrm{p}}-\mathrm{VBM}\right)_{\mathrm{BTO}} .
\end{aligned}
$$

These quantities are determined from the photoemission spectra as detailed in the following.

The Sr $3 d$ spectra recorded on the bare LSMO sample and the BTO/LSMO heterostructure are presented in Fig. 3, and
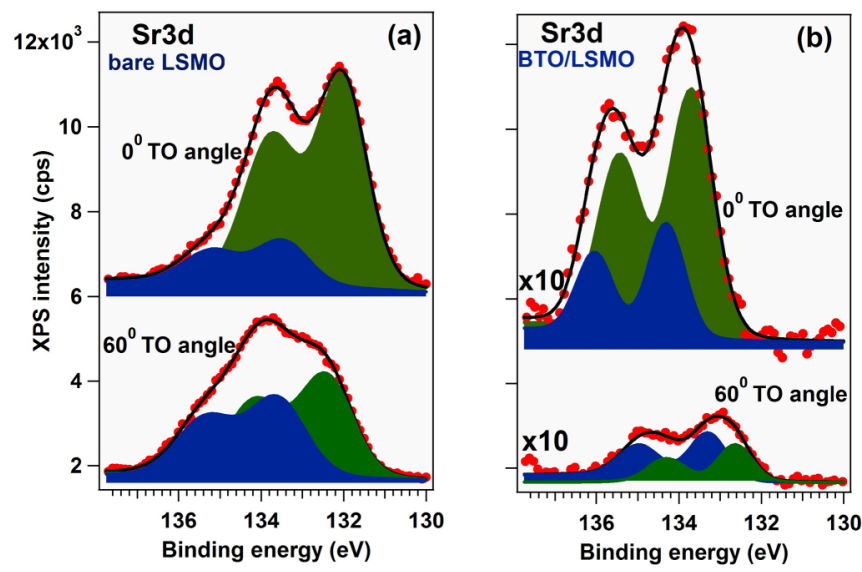

FIG. 3. (Color online) Sr $3 d$ XPS spectra recorded on the two samples at normal incidence and at $60^{\circ}$ (a) on bare LSMO and (b) on BTO-covered LSMO. 

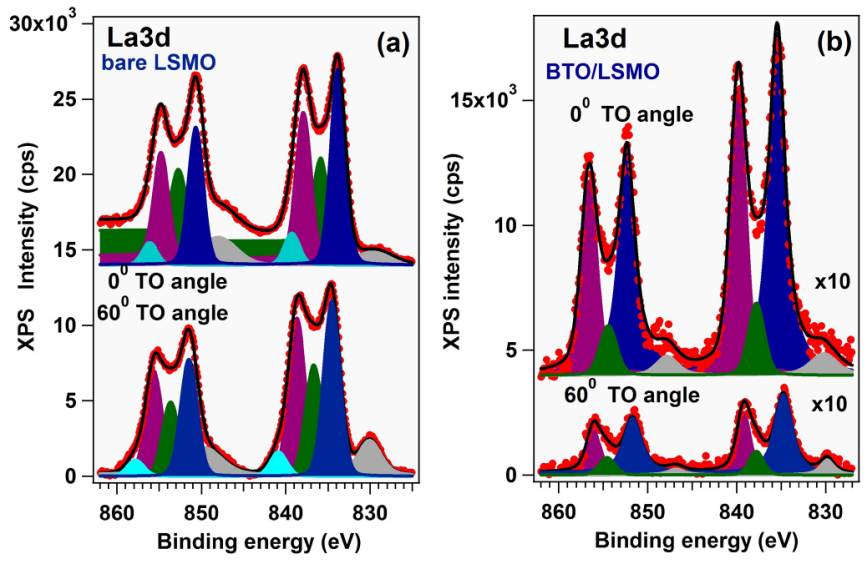

FIG. 4. (Color online) La $3 d$ XPS spectra recorded on the two samples at normal incidence and at $60^{\circ}$ (a) on bare LSMO and (b) on BTO-covered LSMO.

the corresponding La $3 d$ spectra are given in Fig. 4, recorded at normal emission and at a $60^{\circ}$ takeoff angle, in order to resolve the different bulk and surface/interface contributions in the total spectrum. At a $60^{\circ}$ takeoff angle the surface sensitivity is twice that at normal emission. The $\mathrm{Sr} 3 d$ spectra can be satisfactorily described by two doublets. The higher binding energy (HBE) component is twice as intense with respect to the low binding energy (LBE) component at $60^{\circ}$ emission and is shifted by $1.5 \mathrm{eV}$. It therefore represents $\mathrm{Sr}$ at the surface of the LSMO, whereas the LBE component is due to Sr emission from the bulk of the film. The FWHM of $\mathrm{Sr} 3 d_{3 / 2}$ and $\mathrm{Sr}$ $3 d_{5 / 2}$ components is $1.1 \mathrm{eV}$, similar to that previously reported [41-45].

Table I presents the positions of the $\mathrm{Sr} 3 d_{5 / 2}$ components in both bare LSMO and the BTO/LSMO heterostructure for spectra recorded in normal emission. The bulk-related component in the $\mathrm{Sr} 3 d$ spectrum, upon BTO growth, shifts from 132.09 to $133.59 \mathrm{eV}\left(\Delta_{\mathrm{BE}}=1.50 \mathrm{eV}\right)$, while the surface/interface component goes from 133.59 to $134.38 \mathrm{eV}$ $\left(\Delta_{\mathrm{BE}}=0.79 \mathrm{eV}\right)$. The separation between the bulk and surface component changes from $1.5 \mathrm{eV}$ in bare LSMO to $0.79 \mathrm{eV}$ in the BTO/LSMO junction. As seen in Table II, upon BTO growth, the main component of the La $3 d$ spectrum also shifts from 833.64 to $835.26 \mathrm{eV}\left(\Delta_{\mathrm{BE}}=1.62 \mathrm{eV}\right)$.

We now discuss the origin of the $\mathrm{Sr} 3 d \mathrm{HBE}$ component. It is unlikely to be due to $\mathrm{Sr}$ segregated in the form of $\mathrm{SrO}$ as the core-level shift between $\mathrm{Sr} 3 d$ in the perovskite environment and in $\mathrm{SrO}$ is reported to be only $0.2-0.3 \mathrm{eV}$ [46]. Both AFM images and the LEED pattern suggest robust smoothness and crystallinity of the surface, whereas $\mathrm{SrO}$ often segregates in the

TABLE I. Binding energies of the $\mathrm{Sr} 3 d_{5 / 2}$ peak in bare LSMO and BTO-covered LSMO.

\begin{tabular}{lcc}
\hline \hline Sr $3 d_{5 / 2}$ & $\begin{array}{c}\text { Bulk component } \\
(\mathrm{eV})\end{array}$ & $\begin{array}{c}\text { Surface/interface } \\
\text { component }(\mathrm{eV})\end{array}$ \\
\hline LSMO & $132.09 \pm 0.05$ & $133.59 \pm 0.07$ \\
$\mathrm{BTO} \mid \mathrm{LSMO}$ & $133.59 \pm 0.06$ & $134.38 \pm 0.08$ \\
$\Delta \mathrm{E}(\mathrm{eV})$ & 1.50 & 0.79 \\
\hline \hline
\end{tabular}

TABLE II. Binding energies of the La $3 d_{5 / 2}$ peak in bare LSMO and BTO-covered LSMO.

\begin{tabular}{lcc}
\hline \hline La $3 d_{5 / 2}$ & $\begin{array}{c}\text { Bulk component } \\
(\mathrm{eV})\end{array}$ & $\begin{array}{c}\text { Surface/Interface } \\
\text { component }(\mathrm{eV})\end{array}$ \\
\hline LSMO & $833.60 \pm 0.04$ & $838.90 \pm 0.08$ \\
$\mathrm{BTO} \mid \mathrm{LSMO}$ & $855.0 \pm 0.06$ & $839.8 \pm 0.09$ \\
$\Delta \mathrm{E}(\mathrm{eV})$ & 1.7 & 0.9 \\
\hline \hline
\end{tabular}

form of nanostructures. Finally, even in the case of a complete SrO layer on top of the LSMO surface, the contribution of the oxide layer in the total $\mathrm{Sr} 3 d$ spectrum, proportional to $1-$ $\exp \left(-c_{\mathrm{SrO}} / \Lambda \cos \theta\right), c_{\mathrm{SrO}}=5.16 \AA$, would give an intensity ratio $I_{\mathrm{HBE}} / I_{\mathrm{LBE}}$ of approximately 0.66 , more than twice the experimentally observed value of $\sim 0.3$.

The HBE Sr $3 d$ component is therefore most likely due to surface $\mathrm{Sr}$ in the LSMO. In order to assess quantitatively the localization of the XPS surface contribution, an analysis based on the inelastic mean free path (IMFP) has been performed for both BTO and LSMO surfaces. We assumed that each layer of thickness $c$ in the heterostructure attenuates the signal from underlying layers by $\exp (-c / \Lambda \cos \theta)$, with $c$ being the lattice constant in the [001] direction and $\Lambda$ being the inelastic mean free path of the photoelectron at the corresponding kinetic energy. For $\mathrm{Sr} 3 d$ we assumed $\Lambda_{\mathrm{Sr}}=4.8 \mathrm{~nm}$ [47]. From a layer-by-layer attenuation model [48], we derive the fraction $f$ of the total $\mathrm{Sr} 3 d$ spectrum due to surface $\mathrm{Sr}$ atoms considering a (La, Sr)O-terminated surface $f=0.328$, which, compared to the experimental value $f=0.311$, suggests that the LSMO surface is mainly (La,Sr)O terminated. Although La $3 d$ and Mn $2 p$ core electrons have comparable escape depths of 1.6$1.9 \mathrm{~nm}$ [47], the La $3 d$ cross section is more than three times that of $\mathrm{Mn} 2 p$; furthermore, if the $\mathrm{MnO}_{2}$ is below the ( $\left.\mathrm{Sr}, \mathrm{La}\right) \mathrm{O}$ layer, then it will be more strongly attenuated. Thus, the Mn $2 p$ emission is practically absent from the spectra recorded for buried LSMO at a $60^{\circ}$ takeoff angle (Fig. 5).

At the LSMO surface, the $1.5 \mathrm{eV}$ higher-binding-energy component of the $\mathrm{Sr} 3 d$ spectra may be explained by the

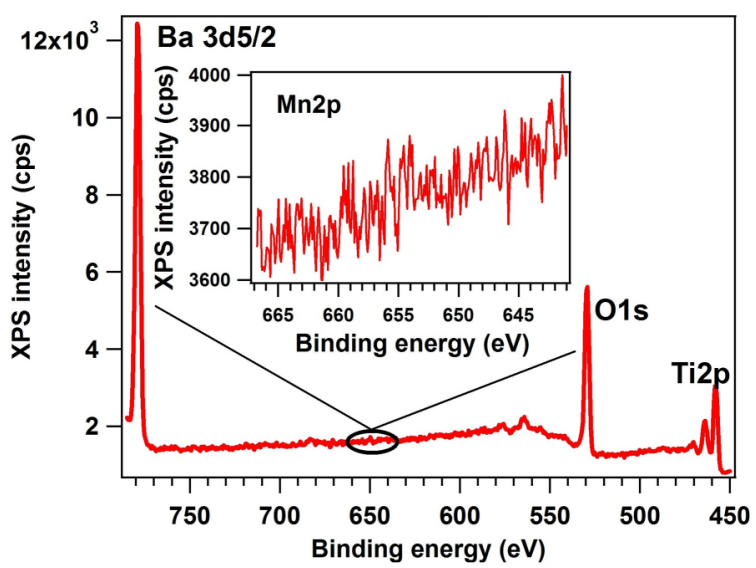

FIG. 5. (Color online) The survey spectra recorded at a normal takeoff angle for the BTO/LSMO heterostructure, with the inset showing the Mn $2 p$ range. 
synergy of two different mechanisms: (i) a dipolar field originating in the positively charged surface with ( $\mathrm{La}, \mathrm{Sr}) \mathrm{O}$ polar termination and (ii) surface rumpling triggered by the buckling of the topmost atomic layers. The former, in principle, operates over a distance comparable to the Thomas-Fermi length, which in LSMO is $\sim 0.3 \mathrm{~nm}$, less than the lattice constant [49], while the latter, associated with off-centering of central $\mathrm{Mn}$ atoms, can propagate into the bulk over longer distances, for approximately 2-3 unit cells, generating a built-in potential similar to the ferroelectric case [50].

The main line (blue) in La $3 d$ spectrum (Fig. 4) can be ascribed to $3 d^{9} 4 f^{0}$ occupation, while the satellite line can be attributed to a charge transfer $3 d^{9} 4 f^{1} L$ (with $L$ representing a hole in a ligand site), corresponding to bonding (cyan) and antibonding (green) satellites. The La $3 d$ spin-orbit splitting is approximately $16.9 \mathrm{eV}$. Based on the variation of the La $3 d$ intensities in normal emission and at $60^{\circ}$, we tentatively ascribe the additional magenta component to a surface/interface atom contribution and the gray peak to a shakedown satellite [51]. The position of La $3 d$ core levels for the LSMO and BTO/LSMO are consistent with results previously obtained by other groups $[52,53]$.

From the integrated XPS intensities recorded in normal emission and the corresponding atomic sensitivity factors [54] we deduce the stoichiometry of the LSMO layer to be $\mathrm{La}_{0.61} \mathrm{Sr}_{0.39} \mathrm{Mn}_{1.04} \mathrm{O}_{2.8}$. This corresponds to an excess hole doping due to $\mathrm{Sr}$ atoms with respect to the $x=0.3$ target and a mild oxygen deficiency. A simple evaluation based on the nominal charges of $\mathrm{Sr}(+2)$ and $\mathrm{O}(-2)$ indicates that the LSMO surface is slightly charge depleted, with a net positive excess charge of $\sim 0.3 e$ per unit cell with respect to the source target. It has been shown that the most favorable vacancy site is in the ( $\mathrm{La}, \mathrm{Sr}) \mathrm{O}$ layer, meaning that we probably have an unaltered $\mathrm{MnO}_{2}$ layer and oxygen-deficient $\left(\mathrm{La}_{0.6} \mathrm{Sr}_{0.4}\right) \mathrm{O}_{0.8}[55]$.

We have used the ratio of the $\mathrm{Sr} 3 d$ intensity $I_{0}$ recorded on bare LSMO to that attenuated by the BTO, $I=I_{0} \exp \left(-d / \Lambda_{\mathrm{Sr}}\right)$, to deduce the thickness of the BTO layer $d=4.2 \mathrm{~nm}$. The difference of $0.8 \mathrm{~nm}$ ( 2 unit cells) between the BTO thicknesses extracted from the XPS intensities and the value assumed from RHEED oscillations possibly comes from an underestimation of deposition time established during the growth calibration.

For the Ti $2 p$ spectra [Fig. 6(a)] the main component is associated with $\mathrm{Ti}$ ions in a perovskite environment with a formal $4+$ valence state, and the smaller component at LBE is classically attributed to reduced $\mathrm{Ti}$ in a $3+$ valence state due to the presence of oxygen vacancies near the BTO surface [56-58]. At the interface of LSMO and strontium titanate, charge transfer from the polar $\left(\mathrm{La}_{1-x} \mathrm{Sr}_{x}\right) \mathrm{O}$ surface to the interface titanate layer has been reported [59], leading to a change in the $\mathrm{Ti}$ valence. The ratio between $\mathrm{Ti}^{4+}$ atoms and the reduced $\mathrm{Ti}^{3+}$ species is a measure of the $n$-type doping in the BTO layer. It was shown recently that ferroelectricity survives to charge doping up to concentrations as high as $1.3-1.9 \times 10^{21} \mathrm{e} / \mathrm{cm}^{3}$, corresponding to approximately $0.09-$ $0.11 e$ /unit cell $[60,61]$. For values higher than this threshold, the free charges quench the ferroelectricity. In our work, the ratio between $\mathrm{Ti}^{3+}$ and $\mathrm{Ti}^{4+}$ is about 0.1 , similar to the findings
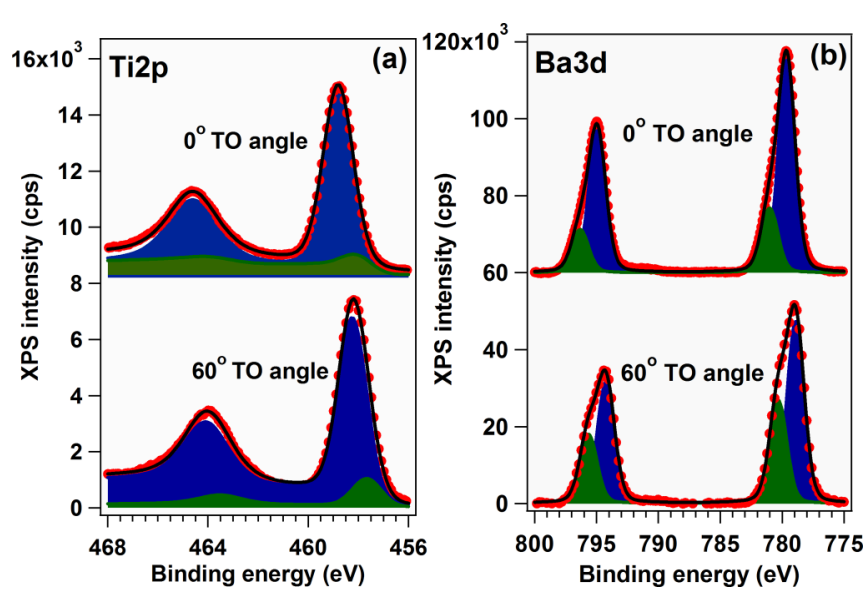

FIG. 6. (Color online) XPS spectra of BTO recorded in the (a) Ti $2 p$ and (b) Ba $3 d$ spectral regions. Top spectra are recorded at normal incidence, and bottom ones are recorded with the samples tilted at $60^{\circ}$ in order to separate the surface and bulk contributions.

reported in Ref. [57]; thus, the ferroelectric state of the BTO layer is assumed to be preserved.

Figure 6(b) shows the two-component $\mathrm{Ba} 3 d$ spectrum recorded at takeoff angles of $0^{\circ}$ and $60^{\circ}$. The spin-orbit split component at LBE was assigned to $\mathrm{Ba}$ atoms in the perovskite phase, and that at HBE was assigned to surfacerelated emission, which has been assigned to undercoordinated surface $\mathrm{Ba}$ atoms [62,63].

Using a similar layer-by-layer attenuation model as in the case of bare LSMO surface and comparing the experimental surface contribution to the whole $\mathrm{Ba} 3 d$ intensity $(f=0.233)$ with the $\mathrm{BaO}$-terminated surface scenario $(f=0.234)$, we confirm that indeed BTO is $\mathrm{BaO}$ terminated. For the $\mathrm{TiO}_{2}$ terminated surface, $\Lambda_{\mathrm{Ti}}=2.4 \mathrm{~nm}$, giving an experimental fraction $f=0.28-0.29$, which is not the case in our work. Thus, it is plausible that undercoordinated $\mathrm{Ba}$ atoms at the BTO surface are at the origin of the higher-binding-energy component in the $\mathrm{Ba} 3 d$ spectrum. Ba $3 d$ also shows a HBE component at the interface with an electrode [39]; however, given the IMPF of approximately $3 \mathrm{~nm}$ and the BTO thickness of $4.2 \mathrm{~nm}$, it is unlikely to make a significant contribution compared to the $\mathrm{Ba} 3 d$ from the bulk of the BTO film.

\section{Schottky barrier height}

Now, we can calculate the Schottky barrier height using Eqs. (1) and (2).

We recall that the $\mathrm{Ti} 2 p$ core levels in the FE layer are characterized by a $0.7 \mathrm{eV}$ shift towards LBE as we depart from the interface with LSMO, in the surface-sensitive measurement (Table III). The value of the Ti $2 p$ shift is consistent with the variation of the valence-band maximum in the interface and surface-sensitive measurements. This variation is the consequence of the depolarization field occurring in the upper layer with the FE polarization pointing downwards due to imperfect screening of the bottom electrode $V(z)$. This is responsible for the skewed band structure near the interface with LSMO, as seen in Fig. 7.

In fact, it was shown previously that when ultrathin ferroelectric layers are grown on a hole-doped ferromagnet 
TABLE III. Binding energies of $\mathrm{Ba} 3 d_{5 / 2}$ and $\mathrm{Ti} 2 p_{3 / 2}$ peaks in the BTO layer for volume and surface-sensitive measurements.

\begin{tabular}{ccc}
\hline \hline Ba $3 d_{5 / 2}$ & $\begin{array}{c}\text { Bulk component } \\
(\mathrm{eV})\end{array}$ & $\begin{array}{c}\text { Surface component } \\
\text { component }(\mathrm{eV})\end{array}$ \\
\hline Normal emission & $779.60 \pm 0.02$ & $781.0 \pm 0.02$ \\
$60^{\circ}$ takeoff angle & $779.00 \pm 0.08$ & $780.1 \pm 0.05$ \\
$\mathrm{Ti} 2 p_{3 / 2}$ & Bulk component & $\begin{array}{c}\text { Surface component } \\
\text { component }(\mathrm{eV})\end{array}$ \\
\hline Normal emission & $458.8 \pm 0.01$ & $458.2 \pm 0.07$ \\
$60^{\circ}$ takeoff angle & $458.2 \pm 0.02$ & $457.6 \pm 0.08$ \\
\hline \hline
\end{tabular}

$\left(\mathrm{La}_{1-x} \mathrm{Sr}_{x} \mathrm{CoO}_{3}\right.$ or LSMO) substrate, the $\mathrm{FE}$ polarization points downwards, whereas when PZT or BTO is grown, for example, on $\mathrm{SrRuO}_{3}$, the $\mathrm{FE}$ polarization points away from the interface [21,24,64-67].

The value of $\Delta V(z)$, the depolarization potential, is extracted from the valence-band spectra of the LSMO/BTO sample taken at normal emission and at a $60^{\circ}$ takeoff angle, resulting in $\Delta V(z)=0.7 \mathrm{eV}$.

The difference between $\mathrm{Ti} 2 p_{3 / 2}$ and $\mathrm{Sr} 3 d_{5 / 2}$ core levels has been extracted from the XPS measurement on the heterostructure; $\left(E_{\mathrm{Sr}}-E_{\mathrm{F}}\right)_{\mathrm{LSMO}}$ has been taken from the measurement performed on the bare LSMO sample and the last term from one previous measurement on a 250 -nm-thick crystalline BTO sample, leading to $\Delta E_{v}=1.28 \mathrm{eV}$.

The general mechanism underlying the band alignment at the interface in the presence of out-of-plane polarization pointing towards the LSMO is presented schematically in Fig. 7 by the solid red line, while with dashed gray line

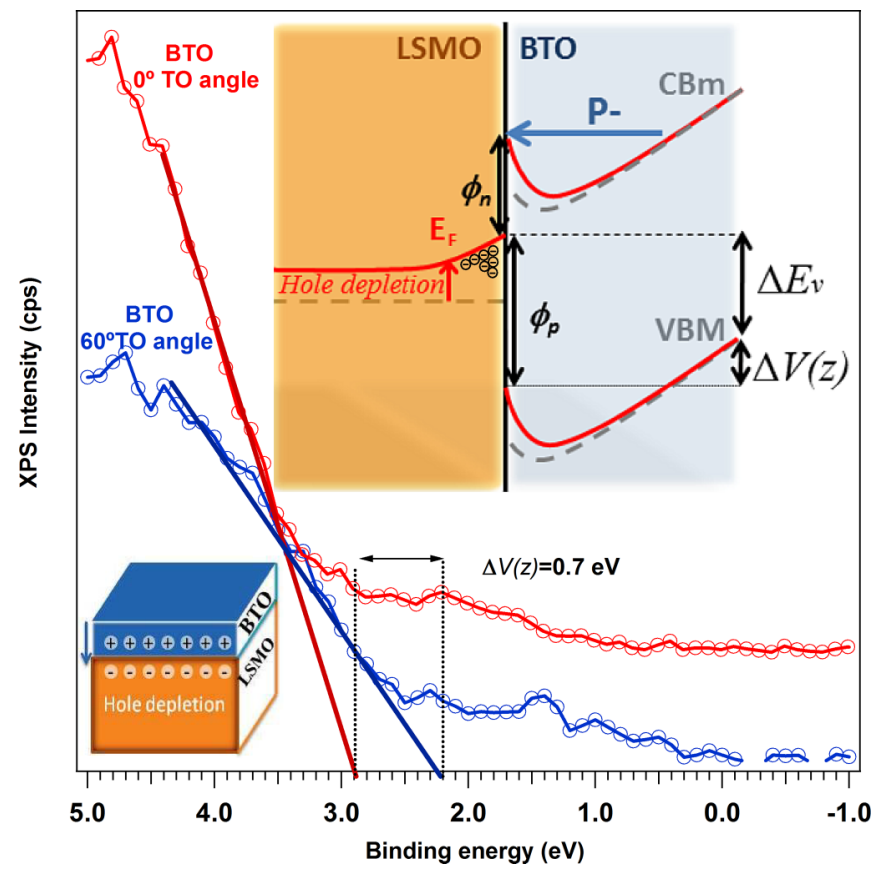

FIG. 7. (Color online) Valence-band region of the LSMO/BTO heterostructure recorded at two collecting angles in order to infer the effects of the built-in potential in the BTO on the valence-band maximum. The inset shows the band alignment and Schottky barrier formation. indicates the situation without the effects of hole depletion at the interface.

The experimental value for the hole barrier height obtained is $\Phi_{p}=1.98 \mathrm{eV}$, including the effects of both interface formation and the depolarization field. If one assumes an experimental BTO band gap of $3.2 \mathrm{eV}$, this corresponds to a Schottky barrier height for electrons of $1.22 \pm 0.17 \mathrm{eV}$ for $P^{-}$polarization.

\section{Hole-depletion effects at the interface}

In the case of 2-5-nm-thick BTO layers, characterized by a single FE domain state over the entire sample, the FE polarization stabilizes towards the interface. We thus expect that the XPS spectra recorded on our LSMO/BTO buried interface reflect the effect of hole depletion on the core-level binding energy. On the other hand, the effects associated with orbital polarization at the interface are not expected to be reflected in XPS measurements; additional x-ray absorption studies are required to observe them.

As can be seen from Tables I and II, there is an appreciable shift towards higher binding energy in both the $\mathrm{Sr} 3 d$ and La $3 d$ main bulk-related components upon BTO deposition. This suggests that a gradual upshift of the Fermi level as a result of the hole-depletion effect near the interface associated with the downwards FE polarization is involved. The shift depends on the exponentially decreasing hole-depletion state induced in the first LSMO u.c. by the FE polarization pointing towards the interface. The variation of the hole distribution within the LSMO slab is controlled by the Thomas-Fermi screening length; thus, the width of the depletion layer may be estimated to approximately 3 u.c. within the LSMO electrode, with the maximum in the first interface layer [68]. The holedepletion effect is visible in the smaller shift of the $\mathrm{Sr} 3 d$ interface component towards higher binding energies than the bulk component. Hole depletion means electron enrichment, thus better core-hole screening and, consequently, a shift to LBE. The FM-to-AFM FE polarization-dependent magnetic order switching at the FE/LSMO interface has been addressed in Refs. [8,68-71], and it was found to be triggered by interface hole depletion or accumulation. In this context, this is the experimental evidence of the hole-depletion state associated with FE polarization pointing towards the interface obtained in photoemission.

\section{FIRST-PRINCIPLES CALCULATIONS}

As a starting point, we have checked the position of the conduction-band minimum (CBM) of BTO with respect to the Fermi level of the LSMO slab for the particular case of the $(\mathrm{La}, \mathrm{Sr}) \mathrm{O} \mid \mathrm{TiO}_{2} \mathrm{LSMO} / \mathrm{BTO}$ junction.

Preliminary calculations performed with and without the Hubbard term applied on Ti atoms indicated that $U_{\mathrm{Ti}}=8 \mathrm{eV}$ not only returns a value closer to the experimental BTO band gap but also pushes the CBM upwards enough to avoid a pathological situation with $\phi_{n, p}<0$ [32].

\section{A. Schottky barrier height}

In the Schottky-Mott limit, $\Phi$ is given by the difference between the insulating ionization potential $I_{P}$ and metal work 

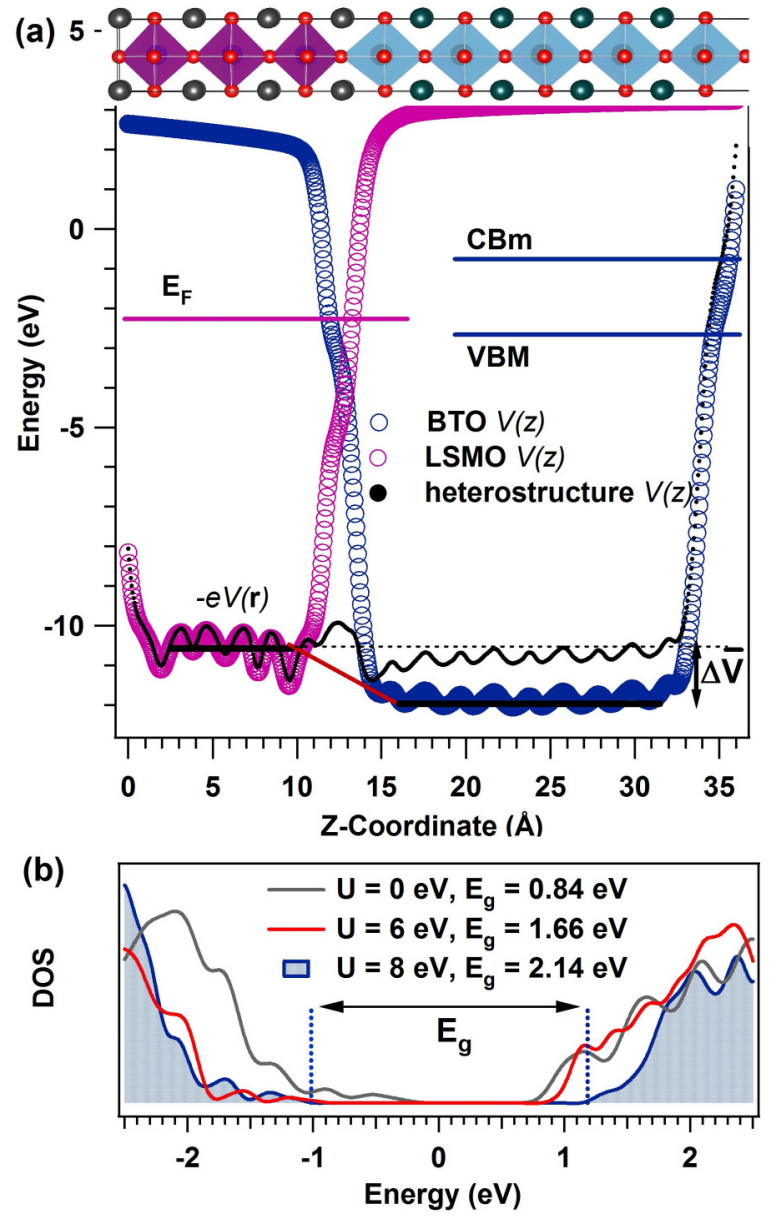

FIG. 8. (Color online) (a) Electrostatic potentials calculated for the LSMO/BTO interface with the top electrode featuring $P^{-}$ polarization. Theoretical band gap for the BTO layer using several values of the Hubbard parameter $U$. (b) For $U=8 \mathrm{eV}$, the conductionband maximum is pushed high enough to prevent charge spillout.

function $W_{F}$. The computations for $I_{P}$ and $W_{F}$ were performed using the same functional and supercells as in the case of band structures and charge densities for both $P^{+}$and $P^{-}$ polarizations. Following the strategy adopted by Berthod et al. [72] and by Zheng and Binggeli [30], we evaluated $I_{P}$ for BTO and $W_{F}$ for LSMO using the following relations:

$$
\begin{gathered}
I_{P}=\Delta V_{\mathrm{BTO}}-\varepsilon_{\mathrm{VBM}}^{\mathrm{BTO}}, \\
W_{F}=\Delta V_{\mathrm{LSMO}}-\varepsilon_{\mathrm{F}}^{\mathrm{LSMO}} .
\end{gathered}
$$

In (3) and (4), $\varepsilon_{\mathrm{VBM}}^{\mathrm{BTO}}$ is the valence-band maximum for the BTO freestanding slab, and $\varepsilon_{\mathrm{F}}^{\mathrm{LSMO}}$ is the Fermi level of the LSMO layer, both evaluated with respect to the average electrostatic potential in the crystal $V(\mathbf{r})$ [see Fig. 8(a)], while $\Delta V_{\mathrm{BTO}}$ and $\Delta V_{\mathrm{LSMO}}$ are the differences between the average electrostatic potential in vacuum and in each of the slabs. $\Delta V_{\mathrm{BTO}}$ and $\Delta V_{\mathrm{LSMO}}$ have been obtained from the macroscopic average across the $x-y$ plane and should, in principle, account for FE polarization-dependent effects, in our case the off-centering of central cations and modulation of bond lengths in the interface region.

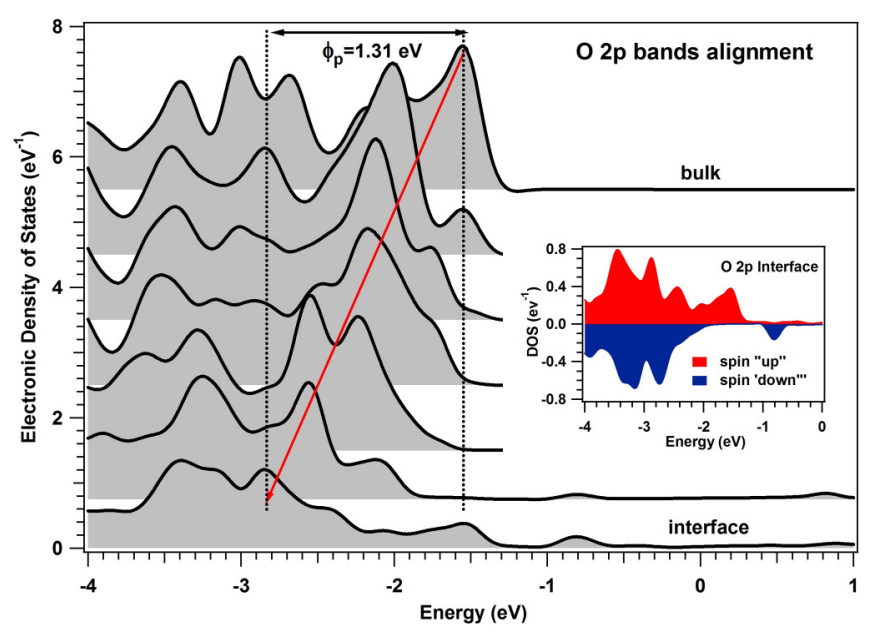

FIG. 9. (Color online) Layer-resolved O $2 p$ electronic density of states for BTO layers from interface towards the bulk. The gradual alignment of the oxygen-derived bands is suggested by the red arrow. The inset presents the spin-resolved DOS of the interface $\mathrm{O} 2 p$ states which are shared by both the LSMO and BTO layers.

With the band terms $E_{\mathrm{F}}$, the Fermi level of LSMO slab; $E_{\mathrm{CBM}}$, the conduction-band minimum; and $E_{\mathrm{VBM}}$, the valence band maximum, all expressed with respect to a reference averaged potential [72] in each crystal [30,73], the theoretical values obtained for the Schottky barrier height as a function of polarization are $\Phi^{\text {down }}=1.37 \mathrm{eV}$ and $\Phi^{\text {up }}=1.03 \mathrm{eV}$. We stress the fact that the calculations were performed for a $(\mathrm{La}, \mathrm{Sr}) \mathrm{O} \mid \mathrm{TiO}_{2}$ junction interface, which is an important observation because previous studies have strongly stressed the importance of the atomic termination on the $\mathrm{SBH}$ [32]. For example, first-principles calculations performed on $\mathrm{LSMO} / \mathrm{SrTiO}_{3}$ heterostructures evidenced differences as high as $2.2 \mathrm{eV}$ between the work functions of $\mathrm{TiO}_{2}$ and SrO-terminated surfaces of $\mathrm{SrTiO}_{3}$ [30]. Here, using a Hubbard parameter $U=8 \mathrm{eV}$ for Ti atoms, although the value calculated for the BTO slab band gap still underestimates the experimental value, the conduction-band minimum is sufficiently pushed away from the Fermi level to avoid charge spillout into the BTO slab and give reliable results on $\phi_{n, p}$. Figure 8(b) illustrates the evolution of the band gap and conduction-band minimum for several values of $U$.

A cross-check of the above observations consists of estimating the Schottky barrier height considering the interface band offset driven by the alignment of interface $\mathrm{O} 2 p$ bands and projecting the layer-resolved density of states on their bulk contribution. The results are displayed in Fig. 9, which indicates that as we approach the interface, a bending of the valence states of $\sim 1.31 \mathrm{eV}$ occurs, which is again in agreement with the results obtained using the surface-averaged electrostatic potential.

The theoretical values $\Phi^{\text {down }}=1.37 \mathrm{eV}$ calculated using the electrostatic potential method and $\Phi^{\text {down }}=1.31 \mathrm{eV}$ obtained using the aligned layer-resolved densities of states are in fair agreement with the experimental value of the SBH of $1.22 \pm 0.17 \mathrm{eV}$. 

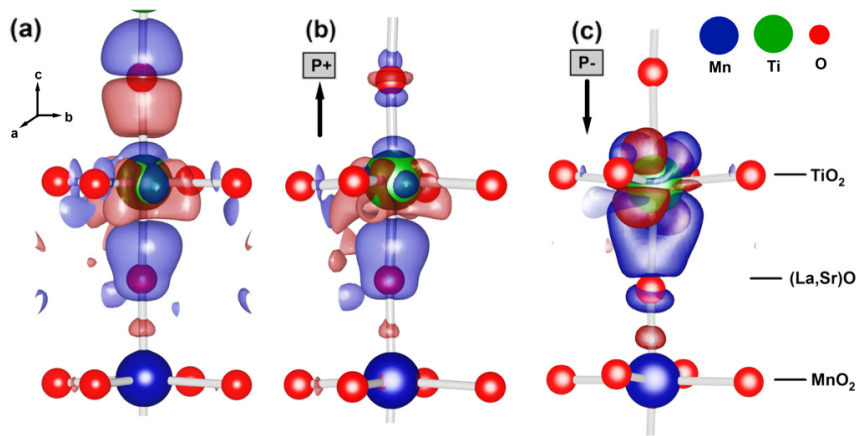

FIG. 10. (Color online) Calculated bonding charge distribution of the LSMO/BTO interface for the paraelectric state: (a) $P=0$, (b) BTO polarization pointing outwards, $P^{+}$, and (c) BTO polarization pointing inwards, $P^{-}$. Red represents the regions with charge depletion, and blue represents the charge-accumulation regions. The isocharge surface is $0.5 e$.

\section{B. Charge-density and spin-dependent orbital states at the interface}

In order to decide whether the interface effects in the LSMO/BTO junction are related to changes in bonding or to electrostatics due to the polarity difference on either side of the interface, the bonding charge distribution for the $(\mathrm{La}, \mathrm{Sr}) \mathrm{O}-\mathrm{TiO}_{2}$ interface has been computed as the difference between the charge density of the heterostructure and that of individual slabs for both out-of-plane polarization directions of BTO. For completeness, the bonding charge [74] has been also calculated for an artificial, tetragonal centrosymmetricparaelectric case. The results are presented in Fig. 10. The main influence of the change in the electronic environment induced by breaking the inversion symmetry at the interface region of the heterostructure along the $c$ axis is observed around the $\mathrm{Ti}$ atoms. For the case studied here of the FE polarization pointing towards the interface, the orbitals involved in the bonding between the LSMO bottom electrode and the top BTO layer have mostly $t_{2 g}(y z)$ symmetry. For the $P^{+}$case, with the FE polarization pointing away from the interface, the bonding is realized through Ti orbitals with in-plane character $-t_{2 g}(x y)$. This suggests an additional contribution of the bonding mechanism to the band lineup at the heterostructure interface.

In previous studies $[2,3,59]$, using a combination of $x$ ray magnetic circular dichroism and transmission electron microscopy, it was shown that at $\left(\mathrm{LaO} \mid \mathrm{TiO}_{2}\right) \mathrm{LaMnO}_{3} / \mathrm{SrTiO}_{3}$ and $(\mathrm{La}, \mathrm{Sr}) \mathrm{O} \mid \mathrm{TiO}_{2} \mathrm{LSMO} / \mathrm{STO}$ interfaces, the $\mathrm{Ti}$ atoms also acquire a finite spin polarization with antiferromagnetic ordering through a superexchange mechanism as a result of hybridization between $d_{x z, y z}$ Ti bonding states and $\mathrm{Mn} e_{g}$ ones, mediated by apical O $p_{z}$ orbitals [4]. Figures 11(a) and 11(b) display the spin-resolved local density of states integrated over $0.2 \mathrm{eV}$ around $E_{\mathrm{F}}$ for FE polarization pointing towards and away from the interface, consistent with this scenario. The total energy difference computed from first principles for two situations [spins with antiferromagnetic (AF) and FM orientation] is $0.9 \mathrm{eV}$, with the $\mathrm{AF}$ ordering being the most stable. That the AF state indeed results from the coupling of $\mathrm{Mn}$ and Ti through the bonding oxygen atom is seen also
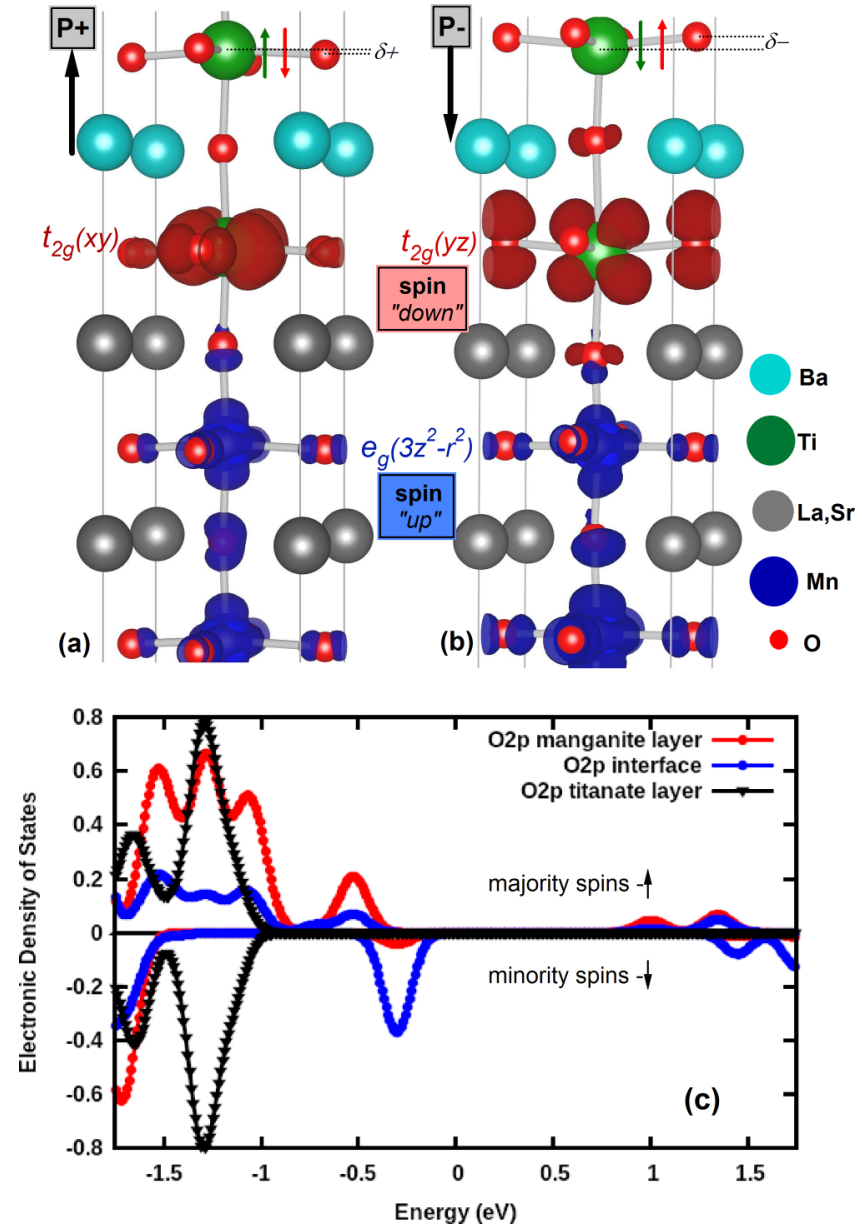

FIG. 11. (Color online) Spin-resolved local density of states integrated $\pm 0.2 \mathrm{eV}$ around $E_{\mathrm{F}}$ for the BTO layer polarization pointing (a) upwards and (b) downwards. Majority up spins are depicted in blue, and minority down spins are in red. The isosurface is $0.004 \mathrm{eV}^{-1}$. (c) The electronic density of states for $\mathrm{O} 2 p$ states in the manganite environment at the interface and in the titanate layer.

in Fig. 11(c), which shows the contribution of $\mathrm{O} 2 p$ apical oxygen atoms to the total density of states in the manganite layer, at the interface, and into the titanate region. Notably, one can clearly identify the peak at $-0.4 \mathrm{eV}$ in the minority-spin channel, which is strongly amplified in the interface region and fades away as we depart from the interface.

In our case, with BTO replacing STO [2,3,59], an additional degree of freedom associated with FE polarization comes into play. It leads to FE-induced charge-density reorganization around $\mathrm{Ti}$ atoms as follows: when the FE polarization of the upper layer points away from the interface, the bonding charge is confined mostly at the $t_{2 g}(x y)$ orbitals, while when the polarization points towards the interface, the states involved in chemical bonding are mostly Ti $t_{2 g}(y z)$ ones. This situation resembles that described in Ref. [17], where at the LSMO/BTO $\left(\mathrm{MnO}_{2} \mid \mathrm{BaO}\right)$ heterojunction, the lifting of degeneracy in the Mn $e_{g}$ orbitals has been noted, accompanied by FE-dependent occupation of $3 r^{2}-z^{2}$ (out-of-plane) or $x^{2}-y^{2}$ (in-plane) states. 
This makes the $\mathrm{MnO}_{2}$ interface layer the active magnetoelectrical "device" via effects associated with variation of the Mn-O bond and thus the change in the hopping integrals $t$ with respect to the paraelectric case $[1,8,17,68,71]$.

On the other hand, for the $\mathrm{TiO}_{2}$ active interface, which has been considerably less studied, our results indicate that in the case of the $(\mathrm{La}, \mathrm{Sr}) \mathrm{O} \mid \mathrm{TiO}_{2}$ interface, the qualitative modifications of the bonding mechanism as a function of the polarization state of the BTO are connected with the variations in the covalent nature of Ti atoms in the Ti-O-Ti/Ti$\mathrm{O}-\mathrm{Mn}$ sequence. As might be expected, the symmetry of the electronic charge distribution is FE polarization sensitive, as seen in Fig. 10. Our results also indicate that $\mathrm{Ti}$ atoms are active in magnetoelectric coupling and stabilize an A-type AF state through different occupations of $t_{2 g}(x y)$ or $t_{2 g}(y z)$ orbitals [2-5,59], depending on the orientation of the FE polarization in conjunction with a superexchange mechanism.

Moreover, the spin-dependent orbital polarization and AF coupling visible in Fig. 11 indicate that the electronic populations at the Fermi level are a combination of spin-up Mn $e_{g}\left(3 z^{2}-r^{2}\right)$ states and spin-down Ti $t_{2 g}(x y)$ ones for $P^{+}$ polarization and spin-up Mn $e_{g}\left(3 z^{2}-r^{2}\right)$ with a Ti $t_{2 g}(y z)$ contribution for the opposite FE polarization of the upper layer. The calculated spin polarization for the interface Ti atoms is $-0.27 \mu_{\mathrm{B}}$ in the $P^{+}$case and $-0.78 \mu_{\mathrm{B}}$ for $P^{-}$polarization.

The orbital polarization at the interface is consistent with the mechanism described in Ref. [17], and it strongly depends on the ratio of the in-plane and out-of-plane lattice constant $c / a$. In our work, for $c / a=0.985<1$ (when the upper layer has $P^{-}$polarization), the orbitals perpendicular to the interface have lower energy, and their occupation is favored $\left(y z\right.$ plane), while in the case of $P^{+}$polarization, $c / a=1.024>1$ and the in-plane orbitals are involved in the bonding mechanism ( $x y$ plane).

A similar coupling mechanism has been invoked by $\mathrm{Yu}$ et al. [5], and our calculated values for the magnetic moments are close to what they measured in $\mathrm{BiFeO}_{3} / \mathrm{La}_{0.7} \mathrm{Sr}_{0.3} \mathrm{MnO}_{3}$ heterostructures, where a significant enhancement of interface Fe magnetic moments from $0.03 \mu_{\mathrm{B}}$ to $\sim 0.6 \mu_{\mathrm{B}}$ has been found. Although the exact value of the spin polarization at the interface is not the central point of this work, we note that the transfer of spin-down density from $x y$ to $y z$ orbitals might be important in modulating the spin-dependent conductance parallel with the heterostructure interface and perpendicular to it by FE switch.

Electronic states with the same symmetry have been previously deduced [30] at the LSMO/STO interface of a non-FE system, but the calculated magnetic moment of $\mathrm{Ti}$ atoms at the interface was considerably smaller $\left(0.1 \mu_{\mathrm{B}}\right)$ than in our case, close to the experimental findings for the same system [59]. It is interesting to note that in a paraelectric case, all Ti $t_{2 g}$ electronic states coexist, and their degeneracy is lifted only in the case of ferroelectric distortion at the interface.

\section{CONCLUSIONS}

The effects associated with the hole-doped $\left(\mathrm{La}_{0.6} \mathrm{Sr}_{0.4} \mathrm{MnO}_{3}\right) / \mathrm{BaTiO}_{3}$ interface formation have been investigated using $\mathrm{x}$-ray photoemission spectroscopy measurements and first-principles calculations. We identified two mechanisms active in the interface band lineup: one residing in the intrinsic electronic effects of the joining materials and another triggered by the ferroelectric polarization. The latter, on the one hand, skews the electronic bands in the interface region due to the depolarizing field and, on the other hand, stabilizes a hole-depletion state in the LSMO, near the contact region. In these conditions, we established that the Schottky barrier height at the (La, $\mathrm{Sr})-\mathrm{O} \mid \mathrm{TiO}_{2}$ interface with the $\mathrm{FE}$ polarization pointing towards the interface is $1.22 \pm 0.17 \mathrm{eV}$.

We also reported the signature in $\mathrm{x}$-ray photoelectron spectroscopy of the hole-depleted state in the LSMO electrode in contact with a ferroelectric, which is responsible for the transition from the ferromagnetic state to the antiferromagnetic state. The signature reveals itself in a distinct lower magnitude of the shifts toward lower binding energies of both $\mathrm{Sr} 3 d$ and La $3 d$ surface core-level components upon a thin ferroelectric layer deposition. This result has an impact on correctly identifying the magnetic phase transition in connection with the ferroelectric activity in the contact region. We examined the differences between the $\mathrm{MnO}_{2} \mid \mathrm{BaO}$ and $(\mathrm{Sr}, \mathrm{La}) \mathrm{O} \mid \mathrm{TiO}_{2}$ interfaces with respect to the mechanisms of orbital polarization and magnetic coupling based on first-principles calculations. Different from the $\mathrm{MnO}_{2} \mid \mathrm{BaO}$ case in which the interface magnetism is triggered by the double-exchange mechanism between $\mathrm{Mn}$ atoms, accompanied by different orbital occupations of $e_{g}$ states for FE polarization pointing towards or away from the interface, at the ( $\mathrm{La}, \mathrm{Sr})-\mathrm{O} \mid \mathrm{TiO}_{2}$ polar interface the degeneracy of the $t_{2} g$ orbitals is lifted depending on the FE polarization, and Ti atoms get a finite spin polarization with antiferromagnetic ordering through a doubleexchange interaction with the Mn atoms through the apical oxygen atoms. These predictions are not directly reflected in the photoemission data, and further x-ray magnetic and linear dichroism in conjunction with spin-resolved photoemission is scheduled to reveal the orbital contribution of the interface bonding mechanism and its dependence on the ferroelectric state.

\section{ACKNOWLEDGMENTS}

This work was supported by the Romanian UEFISCDI funding agency through Contracts No. PN-II-ID-PCCE-20112-0006 and No. PN-II-ID-JRP-2011-2. M.A.H. acknowledges the partial funding from the PN-II-RU-TE-2014-41117 project, and D.G.P. and N.B. acknowledge CEA-IFA MULTIFERRODMS project.
[1] Y. W. Yin, J. D. Burton, A. Y. Borisevich, S. J. Pennycook, S. M. Yang, T. W. Noh, A. Gruverman, X. G. Li, E. Y. Tsymbal, and Q. Li, Nat. Mater. 12, 397 (2013).
[2] J. Garcia-Barriocanal, F. Y. Bruno, A. Rivera-Calzada, Z. Sefrioui, N. M. Nemes, M. Garcia-Hernández, J. Rubio-Zuazo, G. R. Castro, M. Varela, S. J. Pennycook, C. Leon, and J. Santamaria, Adv. Mater. 22, 627 (2010). 
[3] J. Garcia-Barriocanal, J. C. Cezar, F. Y. Bruno, P. Thakur, N. B. Brookes, C. Utfeld, A. Rivera-Calzada, S. R. Giblin, J. W. Taylor, J. A. Duffy, S. B. Dugdale, T. Nakamura, K. Kodama, C. Leon, S. Okamoto, and J. Santamaria, Nat. Commun. 1, 82 (2010).

[4] S. Okamoto, Phys. Rev. B 82, 024427 (2010).

[5] P. Yu, J.-S. Lee, S. Okamoto, M. D. Rossell, M. Huijben, C.-H. Yang, Q. He, J. X. Zhang, S. Y. Yang, M. J. Lee, Q. M. Ramasse, R. Erni, Y.-H. Chu, D. A. Arena, C.-C. Kao, L. W. Martin, and R. Ramesh, Phys. Rev. Lett. 105, 027201 (2010).

[6] M. K. Niranjan, J. D. Burton, J. P. Velev, S. Jaswal, and E. Y. Tsymbal, Appl. Phys. Lett. 95, 052501 (2009).

[7] J. D. Burton and E. Y. Tsymbal, Appl. Phys. Lett. 106, 232904 (2012).

[8] C. A. F. Vaz, J. Hoffman, Y. Segal, J. W. Reiner, R. D. Grober, Z. Zhang, C. H. Ahn, and F. J. Walker, Phys. Rev. Lett. 104, 127202 (2010).

[9] G. Radaelli, D. Petti, E. Plekhanov, I. Fina, P. Torelli, S. R. Salles, M. Cantoni, C. Rinaldi, D. Gutiérrez, G. Panaccione, M. Varela, S. Picozzi, J. Fontcuberta, and R. Bertacco, Nat. Commun. 5, 3404 (2014).

[10] D. Pantel, S. Goetze, D. Hesse, and M. Alexe, Nat. Mater. 11, 289 (2012)

[11] V. Garcia, M. Bibes, L. Bocher, S. Valencia, F. Kronast, A. Crassous, X. Moya, S. Enouz-Vedrenne, A. Gloter, D. Imhoff, C. Deranlot, N. D. Mathur, S. Fusil, K. Bouzehouane, and A. Barthelemy, Science 327, 1106 (2010).

[12] S. Mathews, R. Ramesh, T. Vankatesan, and J. Benedetto, Science 276, 238 (1997).

[13] A. Mardana, S. Ducharme, and S. Adenwalla, Nano Lett. 11, 3862 (2011).

[14] K. M. Rabe, J.-M. Triscone, and C. H. Ahn, Physics of Ferroelectrics: A Modern Perspective (Springer, Berlin, 2007), p. 43.

[15] T. Akimoto, Y. Maruyama, Y. Moritomo, A. Nakamura, K. Hirota, K. Ohoyama, and M. Ohashi, Phys. Rev. B 57, R5594 (1998).

[16] J. H. Park, E. Vescovo, H.-J. Kim, C. Kwon, R. Ramesh, and T. Venkatesan, Nature (London) 392, 794 (1998).

[17] H. Chen, Q. Qiao, M. S. J. Marshall, A. B. Georgescu, A. Gulec, P. J. Phillips, R. F. Klie, F. J. Walker, C. H. Ahn, and S. IsmailBeigi, Nano Lett. 14, 4965 (2014).

[18] C. L. Wu, P. W. Lee, Y. C. Chen, L. Y. Chang, C. H. Chen, C. W. Liang, P. Yu, Q. He, R. Ramesh, and Y. H. Chu, Phys. Rev. B 83, 020103(R) (2011).

[19] N. G. Apostol, L. E. Stoflea, G. A. Lungu, C. Chirila, L. Trupina, R. F. Negrea, C. Ghica, L. Pintilie, and C. M. Teodorescu, Appl. Surf. Sci. 273, 415 (2013).

[20] F. Chen and A. Klein, Phys. Rev. B 86, 094105 (2012).

[21] N. G. Apostol, L. E. Stoflea, G. A. Lungu, L. C. Tanase, C. Chirila, L. Frunza, L. Pintilie, and C. M. Teodorescu, Thin Solid Films 545, 13 (2013).

[22] D. G. Popescu, M. Husanu, L. Trupina, L. Hrib, L. Pintilie, A. Barinov, S. Lizzit, P. Lacovig, and C. M. Teodorescu, Phys. Chem. Chem. Phys. 17, 509 (2015).

[23] M. Husanu, D. G. Popescu, C. Tache, N. G. Apostol, A. Barinov, and C. M. Teodorescu, Appl. Surf. Sci. 352, 73 (2015).

[24] I. Pintilie, C. M. Teodorescu, C. Ghica, C. Chirila, A. G. Boni, L. Hrib, I. Pasuk, R. Negrea, N. Apostol, and L. Pintilie, ACS Appl. Mater. Interfaces 6, 2929 (2014).
[25] C. M. Teodorescu, J. M. Esteva, R. C. Karnatak, and A. El Afif, Nucl. Instrum. Methods Phys. Res., Sect. A 345, 141 (1994).

[26] D. Mardare, D. Luca, C. M. Teodorescu, and D. Macovei, Surf. Sci. 601, 4515 (2007).

[27] P. Giannozzi et al., J. Phys.: Condens. Matter 21, 395502 (2009).

[28] G. Kresse and D. Joubert, Phys. Rev. B 59, 1758 (1999).

[29] J. P. Perdew, K. Burke, and Y. Wang, Phys. Rev. B 54, 16533 (1996).

[30] B. Zheng and N. Binggeli, Phys. Rev. B 82, 245311 (2010).

[31] K. Suzuki and K. Kijima, Jpn. J. Appl. Phys. 44, 2081 (2005).

[32] M. Stengel, P. Aguado-Puente, N. A. Spaldin, and J. Junquera, Phys. Rev. B 83, 235112 (2011).

[33] R. Resta, Ferroelectrics 136, 51 (1992).

[34] J. Dionot, G. Geneste, C. Mathieu, and N. Barrett, Phys. Rev. B 90, 014107 (2014).

[35] T. Hahn, International Tables for Crystallography, Volume A: Space Group Symmetry (Springer, Berlin, 2002).

[36] A. M. Kolpak, D. Li, R. Shao, A. M. Rappe, and D. A. Bonnell, Phys. Rev. Lett. 101, 036102 (2008).

[37] B. C. Huang, Y. T. Chen, Y. P. Chiu, Y. C. Huang, J. C. Yang, Y. C. Chen, and Y. H. Chu, Appl. Phys. Lett. 100, 122903 (2012).

[38] N. G. Apostol, L. E. Stoflea, G. A. Lungu, C. A. Tache, D. G. Popescu, L. Pintilie, and C. M. Teodorescu, Mater. Sci. Eng. B 178, 1317 (2013).

[39] J. E. Rault, G. Agnus, T. Maroutian, V. Pillard, Ph. Lecoeur, G. Niu, B. Vilquin, M. G. Silly, A. Bendounan, F. Sirotti, and N. Barrett, Phys. Rev. B 87, 155146 (2013).

[40] E. A. Kraut, R. W. Grant, J. R. Waldrop, and S. P. Kowalczyk, Phys. Rev. Lett. 44, 1620 (1980).

[41] R. Bertacco, J. P. Contour, A. Barhelemy, and J. Olivier, Surf. Sci. 511, 366 (2002).

[42] A. F. Monsen, F. Song, Z. S. Li, J. E. Boschker, T. Tybell, E. Wahlstrom, and J. W. Wells, Surf. Sci. 606, 1360 (2012).

[43] P. V. Nagarkar, P. C. Searson, and F. D. Gealy, J. Appl. Phys. 69, 459 (1991).

[44] K. Horiba, A. Chikamatsu, H. Kumigashira, M. Oshima, N. Nakagawa, M. Lippmaa, K. Ono, M. Kawasaki, and H. Koinuma, Phys. Rev. B 71, 155420 (2005).

[45] A. Chikamatsu, H. Wadati, H. Kumigashira, M. Oshima, A. Fujimori, M. Lippmaa, K. Ono, M. Kawasaki, and H. Koinuma, Phys. Rev. B 76, 201103(R) (2007).

[46] R. P. Vasquez, J. Electron Spectrosc. Relat. Phenom. 56, 217 (1991).

[47] S. Tanuma, C. J. Powell, and D. R. Penn, Surf. Interface Anal. 21, 165 (1994).

[48] A. Pancotti, J. Wang, P. Chen, L. Tortech, C. M. Teodorescu, E. Frantzeskakis, and N. Barrett, Phys. Rev. B 87, 184116 (2013).

[49] M. Minohara, R. Yasuhara, H. Kumigashira, and M. Oshima, Phys. Rev. B 81, 235322 (2010).

[50] J. M. Pruneda, V. Ferrari, R. Rurali, P. B. Littlewood, N. A Spaldin, and E. Artacho, Phys. Rev. Lett. 99, 226101 (2007).

[51] S.-J. Oh, G.-H. Kim, G. A. Sawatzky, and H. T. Jonkman, Phys. Rev. B 37, 6145 (1988).

[52] M. F. Sunding, K. Hadidi, S. Diplas, O. M. Løvvik, T. E. Norby, and A. E. Gunnæs, J. Electron Spectrosc. Relat. Phenom. 184, 399 (2011).

[53] D. A. Pawlak, M. Ito, M. Oku, K. Shimamura, and T. Fukuda, J. Phys. Chem. B 106, 504 (2002).

[54] C. D. Wagner, L. E. Davis, M. V. Zeller, J. A. Taylor, R. M. Raymond, and L. H. Gale, Surf. Interface Anal. 3, 211 (1981). 
[55] K. Wang, Y. Ma, and K. Betzler, Phys. Rev. B 76, 144431 (2007).

[56] J. D. Baniecki, M. Ishii, T. Shioga, K. Kurihara, and S. Miyahara, Appl. Phys. Lett. 89, 162908 (2006).

[57] S. M. Mukhopadhyay and T. C. S. Chen, J. Mater. Res. 10, 1502 (1995).

[58] K. Morikawa, T. Mizokawa, A. Fujimori, Y. Taguchi, and Y. Tokura, Phys. Rev. B 54, 8446 (1996).

[59] F. Y. Bruno, J. Garcia-Barriocanal, M. Varela, N. M. Nemes, P. Thakur, J. C. Cezar, N. B. Brookes, A. Rivera-Calzada, M. Garcia-Hernandez, C. Leon, S. Okamoto, S. J. Pennycook, and J. Santamaria, Phys. Rev. Lett. 106, 147205 (2011).

[60] X. Liu, Y. Wang, J. D. Burton, and E. Y. Tsymbal, Phys. Rev. B 88, 165139 (2013).

[61] J. E. Rault, J. Dionot, C. Mathieu, V. Feyer, C. M. Schneider, G. Geneste, and N. Barrett, Phys. Rev. Lett. 111, 127602 (2013).

[62] X. L. Li, B. Chen, B. Chen, H. Y. Jing, H. B. Lu, and B. R. Zhao, Appl. Phys. Lett. 87, 222905 (2005).

[63] Y. Fujisaki, Y. Shimamoto, and Y. Matsui, Jpn. J. Appl. Phys. 38, L52 (1999).

[64] A. Gruverman, D. Wu, H. Lu, Y. Wang, H. W. Jang, C. M. Folkman, M. Ye. Zhuravlev, D. Felker, M. Rzchowski, C.-B. Eom, and E. Y. Tsymbal, Nano Lett. 9, 3539 (2009).
[65] J. Chen, H. Lu, J. Liu, Y. H. Chu, S. Dunn, K. Ostrikov, A. Gruverman, and N. Valanoor, Appl. Phys. Lett. 102, 182904 (2013).

[66] E. Arenholz, G. van der Laan, A. Fraile-Rodriguez, P. Yu, Q. He, and R. Ramesh, Phys. Rev. B 82, 140103(R) (2010).

[67] P. Maksymovych, S. Jesse, P. Yu, R. Ramesh, A. P. Baddorf, and S. V. Kalinin, Science 324, 1421 (2009).

[68] X. Ma, A. Kumar, S. Dussan, H. Zhai, F. Fang, H. B. Zhao, J. F. Scott, R. S. Katiyar, and G. Lupke, Appl. Phys. Lett. 104, 132905 (2014).

[69] K. Yoshimatsu, K. Horiba, H. Kumigashira, E. Ikenaga, and M. Oshima, Appl. Phys. Lett. 94, 071901 (2009).

[70] P. M. Leufke, R. Kruk, R. A. Brand, and H. Hahn, Phys. Rev. B 87, 094416 (2013).

[71] J. D. Burton and E. Y. Tsymbal, Phys. Rev. Lett. 106, 157203 (2011).

[72] C. Berthod, N. Binggeli, and A. Baldereschi, Phys. Rev. B 68, 085323 (2003).

[73] P. Rulis, J. Chen, L. Ouyang, W. Y. Ching, X. Su, and S. H. Garofalini, Phys. Rev. B 71, 235317 (2005).

[74] C. Fonseca Guerra, J. W. Handgraaf, E. J. Baerends, and F. M. Bickelhaupt, J. Comput. Chem. 25, 189 (2004). 